\title{
Probing the nucleotide-binding activity of a redox sensor: two-component regulatory control in chloroplasts
}

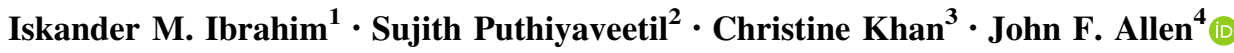

Received: 28 December 2015/Accepted: 2 February 2016/Published online: 12 February 2016

(C) The Author(s) 2016. This article is published with open access at Springerlink.com

\begin{abstract}
Two-component signal transduction systems mediate adaptation to environmental changes in bacteria, plants, fungi, and protists. Each two-component system consists of a sensor histidine kinase and a response regulator. Chloroplast sensor kinase (CSK) is a modified sensor histidine kinase found in chloroplasts-photosynthetic organelles of plants and algae. CSK regulates the transcription of chloroplast genes in response to changes in photosynthetic electron transport. In this study, the fulllength and truncated forms of Arabidopsis CSK proteins were overexpressed and purified in order to characterise their kinase and redox sensing activities. Our results show that CSK contains a modified kinase catalytic domain that binds ATP with high affinity and forms a quinone adduct that may confer redox sensing activity.
\end{abstract}

Keywords Two-component regulatory system - Sensor histidine kinase $\cdot$ Response regulator $\cdot$ Chloroplast sensor kinase - Plastoquinone - Redox regulation - Chloroplast DNA · Transcription - Cyanobacteria $\cdot$ Photosystem stoichiometry

John F. Allen

j.f.allen@ucl.ac.uk

1 Faculty of Engineering and Science, University of Greenwich, Chatham Maritime, Kent, UK

2 Institute of Biological Chemistry, Washington State University, Pullman, WA, USA

312 Ballamaddrell, Port Erin, Isle of Man, UK

4 Research Department of Genetics, Evolution and Environment, University College London, Darwin Building, Gower Street, London WC1E 6BT, UK

\section{Introduction}

Photosynthesis converts light energy from the sun into useful chemical energy. This important biological process takes place in some prokaryotes and in chloroplastsbioenergetic organelles of eukaryotic plants and algae. In photosynthesis, in chloroplasts and cyanobacteria, lightdriven primary electron transfer is carried out by the photochemical reaction centres of two photosystems, photosystem II (PS II) and photosystem I (PS I). A mobile electron carrier-plastoquinone (PQ) - in the thylakoid membrane is a link in the electron transport chain that connects these two reaction centres in series. The reduction-oxidation (redox) state of the pool of PQ molecules determines distribution of excitation energy between PS II and PS I by controlling the reversible phosphorylation of polypeptides of light-harvesting complex II (LHC II) in chloroplasts (Allen 1992; Allen et al. 1981). The redox state of the PQ pool also controls transcription of chloroplast DNA, regulating expression of genes that encode reaction-centre proteins of PS II and PS I, thus initiating a long-term light quality acclimatory process known as photosystem stoichiometry adjustment (Pfannschmidt et al. 1999). In cyanobacteria, prokaryotes from which chloroplasts originated, a similar redox control of photosystem stoichiometry is observed (Fujita 1997; Murakami et al. 1997).

Redox chemistry in the thylakoid membrane is coupled to chloroplast DNA transcription by a bacterial-type twocomponent signal transduction system (TCS). TCSs are the predominant signalling mechanisms in prokaryotic organisms. TCSs consist of two proteins, a sensor histidine kinase (component 1) and a response regulator (component 2) (Stock et al. 2000). Chloroplasts contain a modified bacterial-type sensor histidine kinase, named chloroplast sensor 
kinase (CSK) (Puthiyaveetil et al. 2008). In Arabidopsis, CSK is encoded by the nuclear gene At1g67840 and imported into chloroplasts as a precursor protein synthesised in the cytosol. Under changing light quality conditions that perturb the redox state of the PQ pool, Arabidopsis plants lacking the CSK gene are unable to regulate transcription of the chloroplast reaction-centre gene psaA (encoding the PsaA protein of photosystem I), and are impaired in photosystem stoichiometry adjustments (Puthiyaveetil et al. 2008). CSK is therefore suggested as the sensor component in the signal transduction chain that underlies photosystem stoichiometry adjustments (Puthiyaveetil et al. 2008). The mechanisms by which the photosynthetic electron transport chain controls the activity of CSK, and how CSK regulates the transcription of the psaA gene, are under investigation (Puthiyaveetil et al. 2013). Here, we report on the ATP-binding activity of CSK using the overexpressed, purified full-length recombinant CSK protein. We show that the CSK has a modified ATP-binding site and yet it binds ATP with a $K_{\mathrm{d}}$ value similar to that of unmodified histidine kinases.

\section{Materials and methods}

\section{Secondary structure prediction}

The secondary structure of CSK was predicted using the PSIPRED programme (http://bioinf.cs.ucl.ac.uk/psipred/).

\section{Construction of recombinant plasmids}

Coding sequences for the full-length Arabidopsis CSK protein (CSK_F) and for a truncated version (CSK_T) (amino acids 301 to 611) were amplified from a CSK cDNA clone using primer pairs listed in Table 1. The PCR fragment for CSK_F was digested with NdeI and BamHI endonucleases (New England BioLabs) and cloned into a customised pJC20 expression vector (ATCC). The PCR fragment for CSK_T was cloned into a Gateway pENTR entry vector (Invitrogen) and then mobilised into a customised pETG-10A destination expression vector (EMBL).

Table 1 Primers used for cloning full-length CSK (CSK_F) and truncated CSK (CSK_T). Sequences in lower case are restriction site overhangs

\begin{tabular}{ll}
\hline CSK-F & \\
Forward & GCCGTGcatatgCTTCTTT CTGCAATCGCTTC \\
Reverse & CGAggatccCTATGCTTCATTGGCTTC \\
CSK-T & \\
Forward & CACCATGCAGTCATCTTGGCAAAAC \\
Reverse & CTATGCTTCATTGGCTTC \\
\hline
\end{tabular}

Expression and purification of recombinant CSK protein

Recombinant plasmids were transformed into BL21(DE3) chemically competent cells (stratagene). Transformed bacterial colonies grown on agar plates were used to inoculate starter cultures (20 ml each) in Luria broth (LB) growth medium (8) supplemented with $100 \mu \mathrm{g} \mathrm{mL}^{-1}$ ampicillin as the selection agent. Each culture was grown overnight, then diluted 1:100 in 2 L LB media and grown at $37{ }^{\circ} \mathrm{C}$ to an optical density of $\sim 0.55$ at $600 \mathrm{~nm}$ before inducing protein expression by addition of isopropyl $\beta$-D1-thiogalactopyranoside (IPTG) (Melford) to a final concentration of $100 \mu \mathrm{m}$. Bacterial cultures were grown for a further $16 \mathrm{~h}$ at $17{ }^{\circ} \mathrm{C}$. Cells were harvested by centrifugation at $6000 \mathrm{rpm}$ for $10 \mathrm{~min}$. The pellet was re-suspended in $20 \mathrm{~mL}$ of resuspension buffer $(300 \mathrm{mM} \mathrm{NaCl}, 50 \mathrm{~mm}$ $\mathrm{NaH}_{2} \mathrm{PO}_{4} \mathrm{pH} 7.4,25 \mathrm{~mm}$ imidazole, and $1 \mathrm{~mm}$ PMSF) and the cells lysed with an EmulsiFlex-C3 homogenizer (Avestin). Lysate was separated by centrifugation at $18,000 \mathrm{rpm}$ for $20 \mathrm{~min}$. The supernatant was applied to a $\mathrm{Ni}^{2+}$ affinity chromatography column (GE Healthcare) and the N-terminally poly-histidine tagged CSK protein was purified according to the column manufacturer's instructions. For ATP-binding assay, the elution buffer in the purified protein was exchanged with ATP-binding buffer (10 $\mathrm{mm} \mathrm{NaCl}$ and $10 \mathrm{~mm}$ tris- $\mathrm{HCl}(\mathrm{pH} 8)$ ) using the PD10 desalting column (Amersham Biosciences).

\section{In vitro autophosphorylation assay in the presence of redox agents}

Recombinant CSK protein at $2.5 \mu \mathrm{m}$ concentration was mixed with the kinase reaction buffer $(50 \mathrm{~mm}$ tris- $\mathrm{HCl}$ ( $\mathrm{pH} \mathrm{7.5),} 50 \mathrm{~mm} \mathrm{KCl}, 10 \%$ glycerol, and $10 \mathrm{~mm} \mathrm{MgCl}_{2}$ ) and the following redox agents: $2 \mathrm{~mm} \mathrm{~K}_{3} \mathrm{Fe}(\mathrm{CN})_{6}, 6 \mathrm{~mm}$ DTT, $0.5 \mathrm{~mm}$ benzoquinone, and $0.5 \mathrm{~mm}$ hydroquinone in a final volume of $25 \mu \mathrm{L}$ and incubated at room temperature for $30 \mathrm{~min}$. Autophosphorylation was initiated by the addition of $5 \mu \mathrm{L}$ of 5 -fold concentrated ATP solution giving a final concentration of $0.5 \mathrm{~mm}$ ATP and a specific activity of $5 \mu \mathrm{Ci}\left[\gamma_{-}{ }^{32} \mathrm{P}\right] \mathrm{ATP}\left(6000 \mathrm{Ci} \mathrm{mmol}{ }^{-1}\right)$ (PerkinElmer). Reactions proceeded for $60 \mathrm{~min}$ at $30{ }^{\circ} \mathrm{C}$. The autophosphorylation reaction was stopped by addition of $6 \mu$ of 5X Laemmli sample buffer (Laemmli 1970). Proteins were resolved on a $12 \%$ SDS-PAGE gel, blotted onto a PVDF membrane and the ${ }^{32} \mathrm{P}$-labeling analysed by a phosphor screen.

\section{TNP-ATP-binding assay}

TNP-ATP-binding assay was carried out in a total volume of $3 \mathrm{~mL}$ ATP-binding buffer containing $2 \mu \mathrm{m}$ of CSK_T and 
$1 \mu \mathrm{m}$ of TNP-ATP. Samples were prepared in a $1 \mathrm{~cm} \mathrm{X} 4 \mathrm{~cm}$ quartz cuvette. Fluorescence measurement was carried out using Perkin-Elmer LS55 spectrofluorometer with the excitation wavelength set at $410 \mathrm{~nm}$ and the emission wavelength at 500-650 nm. Excitation and emission monochromators were at $5 \mathrm{~nm}$ and $10 \mathrm{~nm}$ bandwidth, respectively. TNP-ATP was excited at $410 \mathrm{~nm}$ and the fluorescence emission was scanned in a wavelength range of 500-650 nm.

The TNP-ATP-binding titration was carried out by successive addition of varying concentration of TNP-ATP to $2 \mu \mathrm{m}$ CSK_T diluted in ATP-binding buffer. Control titration without protein was performed in the same way. The fluorescence intensity increase at $550 \mathrm{~nm}$ was recorded. Subtracting the control fluorescence value from the sample value corrected for the buffer-TNP-ATP fluorescence. The total volume of TNP-ATP was less than $0.001 \%$ of the total sample volume.

TNP-ATP displacement was carried out by successive addition of varying concentrations of ATP to a sample containing $2 \mu \mathrm{m}$ CSK_T, $1 \mu \mathrm{m}$ TNP-ATP in the ATPbinding buffer. Decrease in fluorescence emission intensity at $550 \mathrm{~nm}$ was monitored. The total volume of ATP added was less than $0.05 \%$ of total sample volume.

Data were analysed as follows: the observed changes in fluorescence at any given concentrations of TNP-ATP ( $\Delta F_{\text {obs }}$ ) were normalised to the total change in fluorescence at infinite concentration $\left(\Delta F_{\text {total }}\right)$, giving rise to the ratio $\frac{\Delta F_{\text {obs }}}{\Delta F_{\text {total }}}$. The The dissociation constant, $K_{\mathrm{d}}$, for TNP-ATP was calculated using Eq. (1):

$\frac{\Delta F_{\text {obs }}}{\Delta F_{\text {total }}}=\frac{\left(K_{d}+L_{t}+E_{t}\right)-\sqrt{K_{d}+L_{t}+E_{t}-4 L_{t} E_{t}}}{2 E_{t}}$,

where $L_{\mathrm{t}}$ is the total ligand concentration (TNP-ATP) and $E_{\mathrm{t}}$ is the total protein concentration.

\section{Results}

\section{Secondary structure prediction of the ATP-lid of CSK}

In order to gain insight into the catalytic domain of CSK, we employed secondary structure prediction. Figure 1 shows the predicted secondary structural elements of cyanobacterial and chloroplast CSKs. With the exception of Cyanidioschyzon merolae CSK, the cyanobacterial and chloroplast CSKs contain a similar secondary structure for the ATP-lid, which is located between the G1 and G2 motifs and is formed of an unstructured coil region. Interestingly, the Cyanidioschyzon merolae CSK contains an unusually large amino acid sequence between the G1 and G2 boxes, perhaps suggesting a larger ATP-lid.

\section{CSK contains a modified CA domain}

The ATP-binding cavity of histidine kinases contains conserved motifs that are essential for their histidine autophosphorylation. These include the G1 and G2 boxes, which have characteristic signatures of "DxGxG" and "GxGxG," respectively. Sequence alignment of CSK homologues shows that plant CSKs have a modified ATPbinding domain (Fig. 2). In higher plants, only the first conserved glycine residue is retained and the second conserved glycine residue in the G1-box is replaced by an aspartic acid (Fig. 2). Moreover, the first two conserved glycine residues in their G2-box are replaced by an asparagine and a valine, respectively. Interestingly, they still retain the third glycine residue in their G2-box (Fig. 2). Some of the algal CSKs, likewise, do not possess a fully conserved G2-motif.

\section{Recombinant protein production}

We successfully cloned the Arabidopsis CSK gene and overexpressed and purified the recombinant protein from E. coli. Figure 3 shows overexpressed and purified fulllength and truncated forms of Arabidopsis CSK. Figure 3a, lane 2 shows the full-length CSK protein being present mainly in the insoluble cell fraction and only in very low abundance in the soluble cell fraction (lane 3). The purified full-length CSK migrated on the SDS-PAGE with an apparent molecular mass of $75 \mathrm{kDa}$. The truncated form of CSK, containing the core kinase domain of CSK, migrates with an apparent molecular mass of $37 \mathrm{kDa}$.

\section{CSK does not autophosphorylate in vitro}

Most protein kinases are autokinase active in that they phosphorylate their own amino acids before phosphorylating their substrates. We therefore examined autophosphorylation activity of full-length CSK in vitro in the presence of different redox agents, which might modulate its kinase activity. The autoradiograph in Fig. 4, lane 1, shows that untreated CSK is inactive as an autokinase. Treatment of CSK with different redox agents did not yield an autokinase active CSK (Fig. 4, lanes 2-5). Interestingly, benzoquinone and hydroquinone-treated CSK proteins migrated as two bands on a $12 \%$ SDS-PAGE (Fig. 4, lane $4,5)$.

\section{CSK binds an ATP analogue TNP-ATP}

Based on sequence alignment shown in Fig. 2, CSK is predicted to contain an ATP-binding cavity, albeit with some modifications. However, the recombinant CSK protein seems to be incapable of an autophosphorylation 


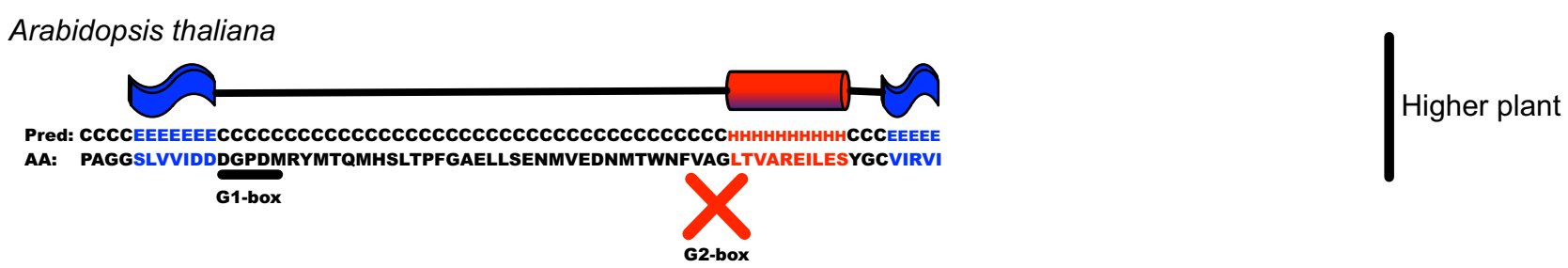

Thalassiosira pseudonana

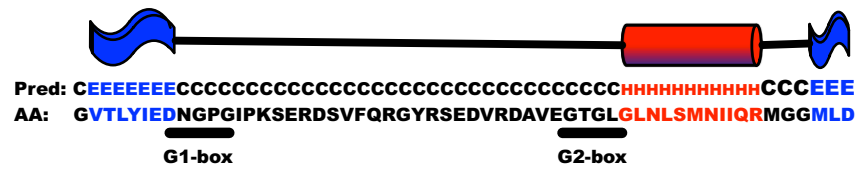

Phaeodactylum tricornutum

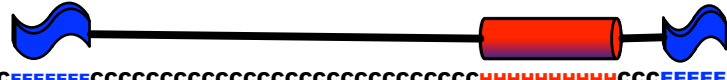

Pred: CCCeefeefeccccccccccccccccccccccCHHHHHHHHHCCCEEEE

AA: PGGGVLIIIEDGDLSTKGFNSARAWRGSDLEYALLGEDFRFVKIVKQEGGVLRVL G1-box

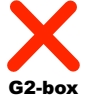

\section{Cyanidioschyzon merolae}

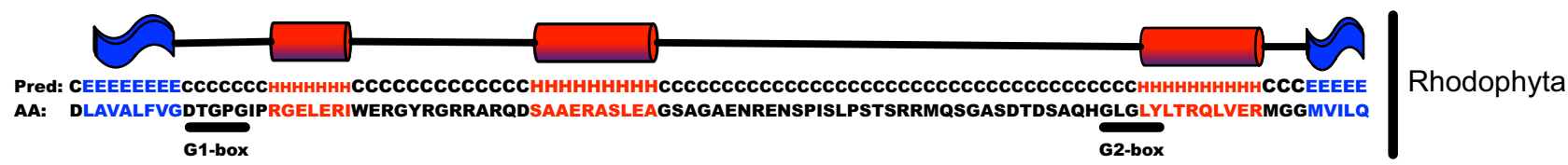

Synechocystis sp. PCC 6803

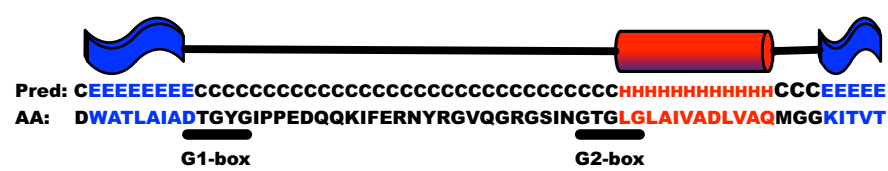

Cyanobacteria

Fig. 1 Predicted secondary structure of the ATP-lid of CSK homologues. Secondary structure of the ATP-lid of cyanobacterial Hik2 and of CSK of Cyanidioschyzon merolae, Thalassiosira pseudonana, Phaeodactylum tricornutum, and Arabidopsis thaliana predicted using the programme PSIPRED (http://bioinf.cs.ucl.ac.uk/psipred/).

reaction in vitro (Fig. 4). In order for CSK to function as a protein kinase, it must have a demonstrable ability to bind an ATP molecule and then catalyse substrate phosphorylation. Here, we investigated the ATP-binding activity of CSK using a fluorescent ATP derivative TNP-ATP. TNPATP contains a trinitrophenyl group at $2^{\prime}\left(3^{\prime}\right)$ hydroxyls of ATP. When exposed to a hydrophobic pocket such as an ATP-binding cavity, TNP-ATP becomes more fluorescent. Figure 5 shows the fluorescence emission spectrum of TNP-ATP in the presence of $2 \mu \mathrm{m}$ CSK (Fig. 5, blue line). The fluorescence emission from TNP-ATP at $550 \mathrm{~nm}$ was increased by more than two-folds in the presence of CSK,
The conserved motifs G1 and G2 boxes are shown. Where the motif is missing, this is indicted by the red cross. AA stands for the target sequence and Pred for the predicated secondary structure. Coil is shown with black line, helix with red oval shape, and strand with blue wave

indicating that TNP-ATP-CSK complex has been formed. The TNP-ATP from CSK can be displaced with the addition of an excess amount of natural ATP (Fig. 5, green line).

\section{TNP-ATP-binding constant}

We next investigated CSK's binding affinity for TNP-ATP by varying the concentration of TNP-ATP. The fluorescence emission increase at $550 \mathrm{~nm}$ was measured and data were fitted to a nonlinear regression model to calculate $K_{\mathrm{d}}$ for TNP-ATP. Figure 6 shows a $K_{\mathrm{d}}$ value of $1 \mu \mathrm{m}$. 


\author{
Arabidopsis thaliana \\ Populus trichocarpa \\ Oryza sativa \\ Physcomitrella patens \\ Micromonas_pusilla_CCMP1545 \\ Ostreococcus lucimarinus \\ Phaeodactylum tricornutum \\ Thalassiosira pseudonana \\ Cyanidioschyzon_merolae \\ Nostoc sp PCC7120 \\ Synechocystis_sp.PCC6803
}

\section{N-Box}

LOVAVEEPALROALSNLIEGALLRTHVGGKVE ILSTRAPAGGSLV LQVAIEEPALROALSNLIEGALLRTHVGGKVEIVSTGAPAGGALV LQVAVEESALRQALSNL I EGALLRTQLGGRVQI YAGEAPAGGILV LHAAVNSASLHRVCSHILETALQHAPRGGYVRANAMRAPGGGVLI ALVAADSRDVHEALALVIDAMLVAAPKGAEVTVVVSAN-KGGVVV TVVYASPKDVRSALAQI IDLALMAAPRGAVIDVAVEEPVQTGVGV PGVTICPQALQEALINVIDNAFTYVFLPGPIRLVQNS-------PGVRACPNYLQEAVSTLLDNAIKYTPIN--VSLLPNK-------PAILADAFALREAISNVLENAIKYTGTVRLIQLVSLNARSAGE IT PLVRVNI KALOEVLSNI IDNALKYTPOGGKIO-----A-----TVVMANRLALREVVNNLLDNGI KYTPNGGLVS-----L-------
G2-Box

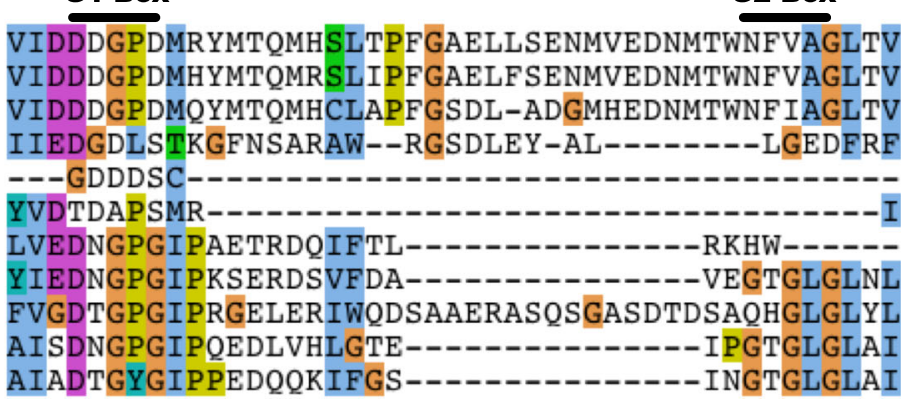

Fig. 2 Conserved sequence features of CA domain of CSK. Sequence alignment of higher plant CSK and its bryophyte, algal, and cyanobacterial homologues. The residue colours scheme is the same as in Fig. 4. The N, G1, and G2 boxes are shown above the sequence

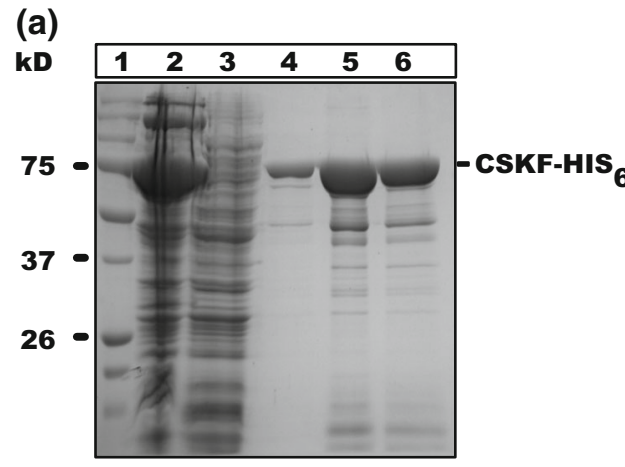

Fig. 3 Protein overexpression and purification. The full-length CSK protein was overexpressed and purified as described in the experimental section. The following samples were loaded on a SDS-PAGE $12 \%$. lane 1 , protein molecular weight marker; lane 2 , total cell

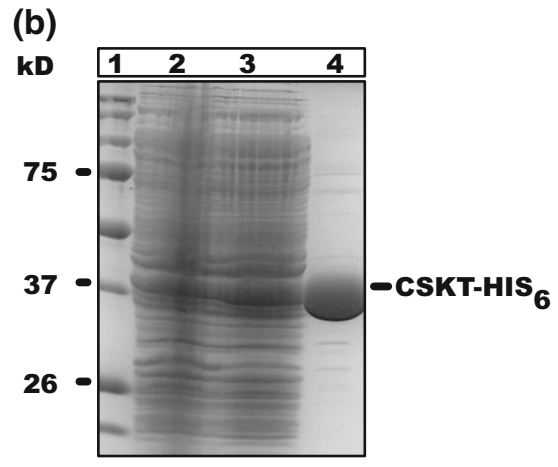

lysate; lane 3, soluble cell fraction; lane 4 and 5 are elution fraction 1. On the left, protein molecular weight markers are shown in $\mathrm{kDa}$. The positions of the overexpressed CSK_F and CSK_T are indicated on the right

\section{TNP-ATP dissociation constant}

We further looked at the competitive exchange of TNPATP with natural ATP. The dissociation constant was determined by titrating CSK with ATP in the presence of $1 \mu \mathrm{m}$ TNP-ATP. The resulting fluorescence decrease of TNP-ATP was measured. Figure 7 shows that a $K_{\mathrm{d}}$ value of $4.97 \mathrm{~mm}$ for ATP can be obtained by fitting the flourescence data using a nonlinear regression model.

\section{Discussion}

A signal transduction cascade by a two-component system is initiated when the histidine kinase, upon sensing an environmental signal, undergoes autophosphorylation in an ATP-dependent manner (Stock et al. 2000). In this study, we provide evidence concerning the nucleotide-binding activity of Arabidopsis CSK. CSK in green algal and land plants has lost the conserved histidine residue required for 


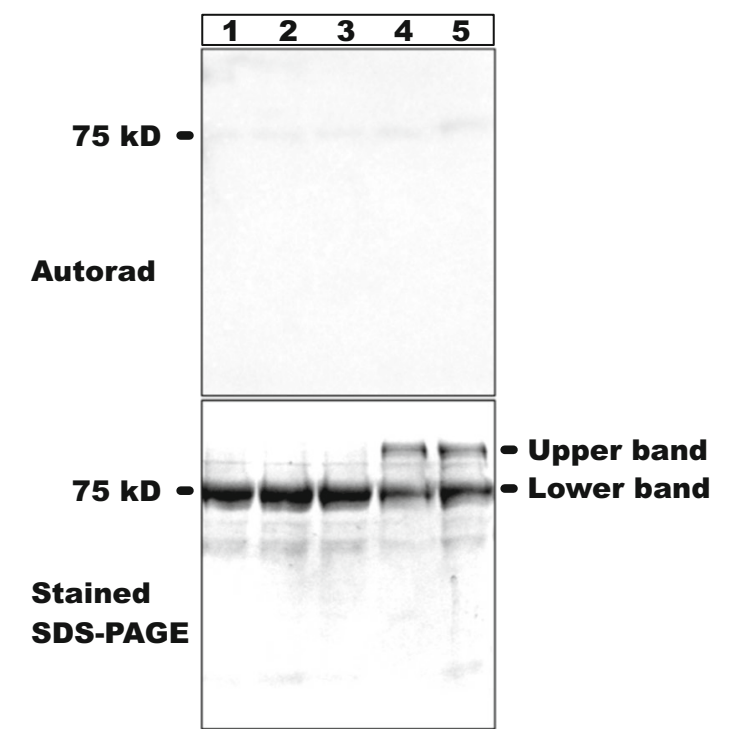

Fig. 4 Effect of different redox agents on the activity of CSK. An autoradiograph of the reaction of CSK proteins pre-treated with lane 1, nothing; lane 2, $2 \mathrm{~mm} \mathrm{~K}_{3} \mathrm{Fe}(\mathrm{CN})_{6}$; lane 3, $6 \mathrm{~mm}$ DTT; lane 4, $0.5 \mathrm{~mm}$ benzoquinone; lane $5,0.5 \mathrm{~mm}$ hydroquinone

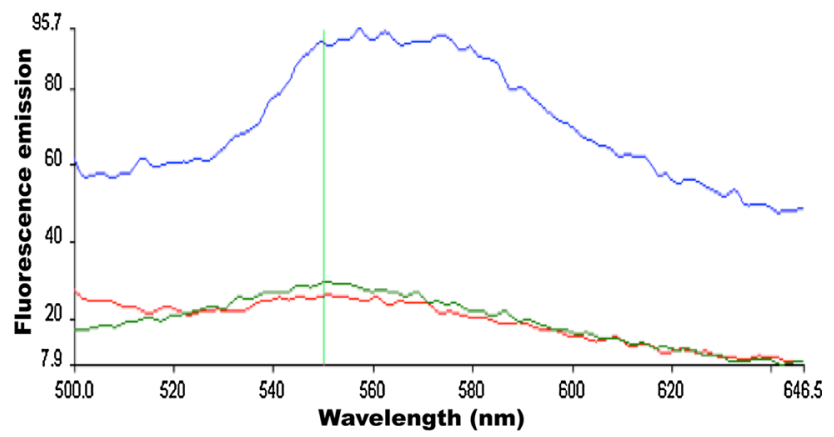

Fig. 5 Binding equilibrium of TNP-ATP to Arabidopsis CSK using fluorescence spectroscopy. TNP-ATP was excited at $410 \mathrm{~nm}$ and excitation emission was measured between 500 and $646 \mathrm{~nm}$. Fluorescence emission spectra of $1 \mu \mathrm{m}$ TNP-ATP is shown by red line; fluorescence spectra of $1 \mu \mathrm{m}$ TNP-ATP in the presence of $2 \mu \mathrm{m} \mathrm{CSK}$ is shown by blue line; and fluorescence spectra of $1 \mu \mathrm{m}$ TNP-ATP in the presence of $2 \mu \mathrm{m}$ CSK and $40 \mathrm{~mm}$ natural ATP is shown by green line

its autophosphorylation and phosphotransfer activities. The conserved histidine residue in CSK has been replaced by a tyrosine or a glutamine in green algae and by a glutamate in higher plants (Puthiyaveetil and Allen 2009; Puthiyaveetil et al. 2008). Correspondingly, there are similar modifications in the CA domain of these modified CSKs (Fig. 1). These modifications, however, does not affect its ATP-binding activity (Figs. 5, 6, 7). The binding affinity of CSK for the fluorescent analogue of ATP (TNP-ATP) is $1 \mu \mathrm{m}$, a value that is comparable to that of bacterial histidine kinases. For TNP-ATP, the EnvZ and CheA kinases have a $K_{\mathrm{d}}$ value of 1.9 and $1.7 \mu \mathrm{m}$, respectively (Table 2)

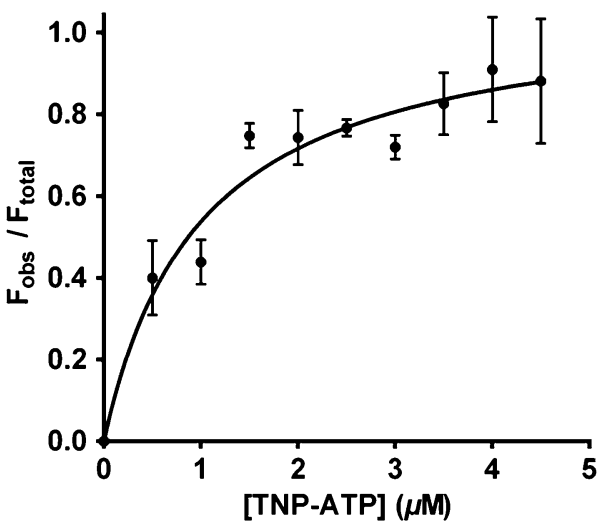

Fig. 6 Titration of Arabidopsis CSK with TNP-ATP. TNP-ATPbinding titration was performed with varying concentration of TNPATP in the presence of $2 \mu \mathrm{m}$ CSK and $1 \mu \mathrm{m}$ ATP. TNP-ATP was excited at $410 \mathrm{~nm}$ and excitation emission at $550 \mathrm{~nm}$ was monitored. Correction to background TNP-ATP fluorescence was made by subtracting values for buffer plus TNP-ATP from CSK plus TNPATP and data were then plotted using prism 5 (Motulsky and Christopoulos 2003). Each data point represents the mean \pm S.E of three measurements. Dissociation constant $\left(K_{\mathrm{d}}\right)$ for TNP-ATP was calculated by nonlinear regression curve fitting of data

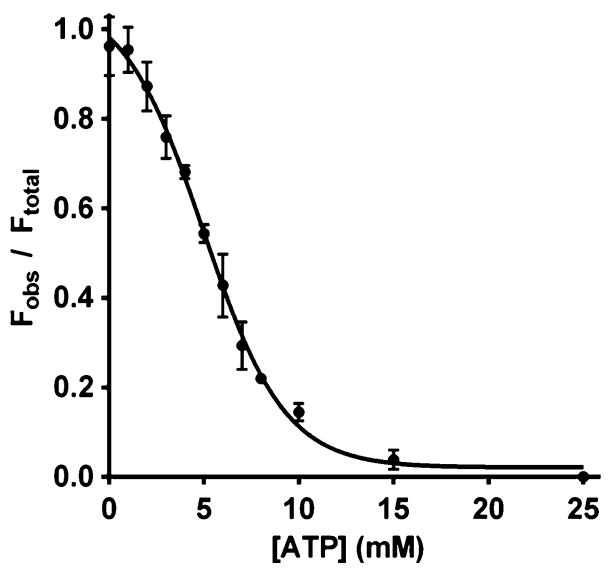

Fig. 7 Displacement of TNP-ATP from Arabidopsis CSK. TNP-ATP displacement titration was performed with varying concentration of ATP to a sample that was pre-equilibrated with $1 \mu \mathrm{m}$ TNP-ATP and $1 \mu \mathrm{m}$ CSK. Decrease in intensity of emission at $550 \mathrm{~nm}$ was measured. Data were plotted as a function of ATP concentration and were plotted using prism 5 (Motulsky and Christopoulos 2003). Each data point represents the mean \pm S.E of three measurements. The dissociation constant $\left(K_{\mathrm{d}}\right)$ for TNP-ATP was calculated by nonlinear regression curve fitting of data

(Plesniak et al. 2002; Stewart et al. 1998). Furthermore, TNP-ATP can be displaced from CSK using unmodified ATP (Fig. 5), implying that TNP-ATP binding has the same characteristics as that of ATP.

The overall structure of the CA domain of the histidine kinase is formed of a $\alpha / \beta$ sandwich fold, composed of three helices and five stranded $\beta$-sheets, which is distinct from that of serine/threonine or tyrosine kinases. However, the 
histidine kinase fold bears strong similarity to the ATPbinding domain of DNA topoisomerase II (Roca and Wang 1992), DNA Gyrase B (Ali et al. 1995), the chaperone Hsp90 (Panaretou et al. 1998), and the DNA repair enzyme MutL (Ban et al. 1999). The CA domain is characteristic of its conserved sequence motifs: N, F, G1, G2, and G3 boxes that are important for stabilisation of an ATP molecule and for the hydrolysis of the ATP $\gamma$-phosphate. The N-box and G1-box are involved in the stabilisation of the adenine ring of ATP. In particular, a conserved aspartic residue found within the G1-box forms a hydrogen bond with the amino N6 of the adenine base (Marina et al. 2001; Trajtenberg et al. 2010). This interaction is crucial for conferring specificity to ATP and to prevent the binding of other nucleotides such as GTP. The CA domains discriminate nucleotides such as GTP based on the fact that they lack the amino group required for forming a hydrogen bond with the carboxyl group of the conserved aspartic acid within the G1-box. The F-box, which is the less conserved region of CA domain, and the G2-box together form a flexible loop that acts as the ATP-lid and controls the entry of the ATP- $\mathrm{Mg}^{2+}$ complex and the release of the ADP- $\mathrm{Mg}^{2+}$ complex. Conserved glycine residues in the G2-box facilitate the flexibility of the ATP-lid (Marina et al. 2001). The G1-box is fully conserved in all CSKs. The G2-box, however, is less conserved in chloroplast CSKs and unrecognisable in higher plants (Fig. 1). Nonetheless, this did not affect their ATP-binding activity. In contrast to higher plant CSK, substitution of any of the glycine residues in the $\mathrm{G} 1$ or $\mathrm{G} 2$ boxes of cyanobacterial CSK results in an inactive kinase (Ibrahim et al. 2016) suggesting that the modified CSKs contain a different catalytic fold.

CSK homologue in cyanobacteria is able to autophosphorylate in vitro and transfer phosphoryl groups to its response regulators Rre1 and RppA. Furthermore, its autokinase activity can be inhibited by sodium ions (Ibrahim et al. 2016). Although the modified CSK binds ATP in vitro (Fig. 5), we could not detect its autophosphorylation (Fig. 4). This could be because of a number of reasons. Firstly, plant CSK could be autokinase inactive at its ground state and thus a signal may be required to activate it. In our assay, this signal may have been absent. Secondly, the CSK might require a cofactor(s) such as FAD for its redox sensing activity and that the recombinant and purified protein used in this study could be an incomplete apoenzyme. A third possibility is that the bacterially expressed recombinant CSK is not folded correctly for the kinase reaction. CSK may well have lost its ability to autophosphorylate and might function in a manner similar to some of the modified bacterial histidine kinases such as DivL. In contrast to DivL, however, CSK exhibits an ATPbinding affinity similar to prototypical histidine kinases, while DivL binds ATP analogue TNP-ATP poorly, with a $K_{\mathrm{d}}$ value of $57 \mu \mathrm{m}$ (Childers et al. 2014). The strong binding affinity of CSK for ATP thus indicates that it has retained its kinase activity.

The lack of a demonstrable autokinase activity in CSK also suggests that the modified CSKs have a catalytic mechanism that differs from that of their cyanobacterial homologues. Perhaps this modification is necessary for CSK to acquire a new substrate, as its cognate response regulators Ycf29 and Ycf27 are missing from chloroplasts in the green lineages (Puthiyaveetil and Allen 2009). Indeed, Arabidopsis CSK protein interacts with plastid transcription machineries, including sigma factor 1 (SIG1) (Puthiyaveetil et al. 2013, 2010), a transcriptional initiation factor that has a prokaryotic origin. SIG1 is required to initiate $p s b A$ and $p s a A B$ transcription (Shimizu et al. 2010).

Histidine kinases do not transfer the $\gamma$-phosphate group directly from an ATP molecule to their substrate. They instead use the high energy of the phosphoramide bond of the phospho-histidine to facilitate the transfer of the phosphate to an aspartate residue of the response regulator. Phospho-serine and -threonine are, however, thermodynamically more stable than phosphoramide or acyl-phosphate, they therefore cannot passively transfer phosphate groups. Indeed, several modified histidine kinases, such as ETR2 (Moussatche and Klee 2004), plant phytochromes (Fankhauser et al. 1999), $\alpha$-ketoacid dehydrogenase kinase (Lasker et al. 2002), and pyruvate dehydrogenase kinase (PDK) (Thelen et al. 2000) have lost their ability to catalyse His-Asp phosphotransfer. They have instead acquired a catalytic mechanism that is similar to that of serine/threonine kinases in that they now phosphorylate their substrates on serine or threonine residues. It is likely that the modified CSKs in green lineage also acquired a serine/threonine-type catalytic mechanism similar to that of modified histidine kinases, a property that is yet to be demonstrated experimentally.
Table 2 Comparison of the binding affinities of the substrates TNP-ATP and ATP to Arabidopsis thaliana CSK, CheA (Stewart et al. 1998) and E. coli EnvZ (Plesniak et al. 2002)

\begin{tabular}{llll}
\hline Sensor histidine kinase & Species & \multicolumn{2}{l}{ Binding affinity } \\
\cline { 3 - 4 } & & TNP-ATP $(\mu \mathrm{m})$ & ATP $(\mathrm{mm})$ \\
\hline CSK & Arabidopsis thaliana & 1.0 & 4.97 \\
CheA & E.coli & 1.7 & 6.0 \\
EnvZ & E.coli & 1.9 & 6.2 \\
\hline
\end{tabular}


The PQ pool has a standard midpoint potential of $+50 \mathrm{mV}, n=2$ (Silverstein et al. 1993b) and an effective redox potential that changes with fluctuating light distribution between the photosystems. These fluctuations may affect a specific redox sensor. For example, the redox state of the PQ pool controls the light-dependent phosphorylation of chloroplast light-harvesting complex II (LHC II) (Allen 1992; Allen et al. 1981). The LHC II kinase (STN7/ STT7) is responsible for phosphorylation of LHC II (Bellafiore et al. 2005; Depege et al. 2003; Rochaix 2007). LHC II kinase has a midpoint potential of $+48 \mathrm{mV}$, which is similar to that of PQ pool midpoint potential (Silverstein et al. 1993a). Some quinone pool sensors, however, do not utilise its redox signal. RegB, for example, interacts with the reduced and oxidised forms of ubiquinone with similar affinities, and only the oxidised form of the ubiquinone seems to switch-off the autophosphorylation of RegB (Wu and Bauer 2010). The autophosphorylation of $\operatorname{RegB}$ is, in fact, regulated through an allosteric effect triggered by binding of ubiquinone/ubiquinol rather than oxidation by ubiquinone (Wu and Bauer 2010). The UV absorption spectra and the primary amino acid sequence of CSK (results not shown) did not reveal potential spectral signatures or binding motifs characteristic of redox sensitive cofactors such as heme or flavin, which suggests that CSK may employ a RegB-type quinone sensing mechanism rather than taking part in direct redox sensing. We also noted that benzoquinone-treated and hydroquinone-treated CSK protein migrated as two bands on the SDS-PAGE gel that is both denaturing and reducing (Fig. 4, lanes 4 and 5). Cysteine residue in enzymes are known to react with quinones to form quinone-cysteine adducts that cannot be broken with reducing agents, for example by DTT used in our sample buffer ( $\mathrm{Li}$ et al. 2005). Cysteine residues in CSK could be forming a thioether-quinone adduct. This observation further strengthens the quinone-binding activity of CSK. However, it is not yet clear whether the adduct formation still occurs with the plastoquinone in the thylakoid membrane.

One question that is still unclear is how a water-soluble redox sensor such as CSK sense the quinone redox signal located within the thylakoid membrane? PQ is a mobile electron carrier that shuttles electrons between PS II and cytochrome $b_{6} f$ in the thylakoid membrane. For membrane-anchored redox sensors, quinone appears to be an attractive candidate as the signalling molecule; however, for soluble redox sensors, it is a more challenging prospect. Nevertheless, there are several soluble proteins that are quinone pool sensors. The NifL sensor protein regulates the $\mathrm{N}_{2}$-fixation (nif) genes in Azotobacter vinelandii (Grabbe and Schmitz 2003). NifL is a flavoprotein that contains a redox active FAD cofactor that is reduced by the quinone pool. The CikA (circadian input kinase A) sensor protein from Synechococcus elongatus PCC 7942 is a soluble protein that senses the PQ pool unaided by a redox-active prosthetic group (Ivleva et al. 2006). In order for CSK to sense the PQ pool, it must have the ability to associate itself with the thylakoid membrane and it must possess a binding site for PQ. For CSK, the ability to associate with the thylakoid membrane may be imparted by the amphipathic helices in its GAF sensor domain. Can the GAF domain in CSK also act as a binding pocket for the reactive, hydrophobic PQ head group? GAF domains are known for their ability to bind small cofactors such as heme and nucleotides (Sardiwal et al. 2005). CSK, indeed, binds DBMIB with a $K_{\mathrm{d}}$ value of $3.66 \mu \mathrm{m}$ (Puthiyaveetil et al. 2013), a binding affinity comparable to other quinone binding proteins and thus strengthening the quinonesensing activity of CSK.

Based on results presented here, we conclude that the catalytic domain of CSK became modified in evolution in order to accommodate a Ser/Thr kinases-type catalytic mechanism that was essential for its incorporation into an existing signal transduction network. CSK's ATP-binding activity and ability to form quinone adducts further strengthen its role in the crucial sensory circuit that connects the redox state of the PQ pool with the chloroplast gene transcription, consistent with the CoRR hypothesis for the evolutionary retention of organellar genomes (Allen 1993a, b, 2003, 2015).

Acknowledgments IMI thanks Queen Mary University of London for a graduate teaching studentship. SP held a Leverhulme Trust early career postdoctoral research fellowship. JFA acknowledges the support of research Grant F/07 476/AQ and fellowship EM-2015-068 of The Leverhulme Trust.

Open Access This article is distributed under the terms of the Creative Commons Attribution 4.0 International License (http://crea tivecommons.org/licenses/by/4.0/), which permits unrestricted use, distribution, and reproduction in any medium, provided you give appropriate credit to the original author(s) and the source, provide a link to the Creative Commons license, and indicate if changes were made.

\section{References}

Ali JA, Orphanides G, Maxwell A (1995) Nucleotide binding to the 43-kilodalton N-terminal fragment of the DNA gyrase B protein. Biochemistry 34:9801-9808

Allen JF (1992) Protein-phosphorylation in regulation of photosynthesis. Biochim Biophys Acta 1098:275-335

Allen JF (1993a) Control of gene-expression by redox potential and the requirement for chloroplast and mitochondrial genomes. J Theor Biol 165:609-631

Allen JF (1993b) Redox control of transcription—sensors, response regulators, activators and repressors. FEBS Lett 332:203-207

Allen JF (2003) The function of genomes in bioenergetic organelles. Philos Trans R Soc Lond B 358:19 
Allen JF (2015) Why chloroplasts and mitochondria retain their own genomes and genetic systems: colocation for redox regulation of gene expression. Proc Natl Acad Sci USA 112:10231-10238

Allen JF, Bennett J, Steinback KE, Arntzen CJ (1981) Chloroplast protein phosphorylation couples plastoquinone redox state to distribution of excitation energy between photosystems. Nature 291:25-29

Ban C, Junop M, Yang W (1999) Transformation of MutL by ATP binding and hydrolysis: a switch in DNA mismatch repair. Cell 97:85-97

Bellafiore S, Barneche F, Peltier G, Rochaix JD (2005) State transitions and light adaptation require chloroplast thylakoid protein kinase STN7. Nature 433:892-895

Childers WS, Xu QP, Mann TH, Mathews II, Blair JA, Deacon AM, Shapiro L (2014) Cell fate regulation governed by a repurposed bacterial histidine kinase. Plos Biol 12

Depege N, Bellafiore S, Rochaix JD (2003) Role of chloroplast protein kinase Stt7 in LHCII phosphorylation and state transition in Chlamydomonas. Science 299:1572-1575

Fankhauser C, Yeh KC, Lagarias JC, Zhang H, Elich TD, Chory J (1999) PKS1, a substrate phosphorylated by phytochrome that modulates light signaling in Arabidopsis. Science 284:15391541

Fujita Y (1997) A study on the dynamic features of photosystem stoichiometry: accomplishments and problems for future studies. Photosynth Res 53:83-93

Grabbe R, Schmitz RA (2003) Oxygen control of nif gene expression in Klebsiella pneumoniae depends on NifL reduction at the cytoplasmic membrane by electrons derived from the reduced quinone pool. Eur J Biochem 270:1555-1566

Ibrahim IM, Puthiyaveetil S, Allen JF (2016) A two-component regulatory system in transcriptional control of photosystem stoichiometry: redox-dependent and sodium ion-dependent phosphoryl transfer from cyanobacterial histidine kinase Hik2 to response regulators Rre1 and RppA. Frontiers in plant science 7 doi:10.3389/fpls.2016.00137

Ivleva NB, Gao TY, LiWang AC, Golden SS (2006) Quinone sensing by the circadian input kinase of the cyanobacterial circadian clock. Proc Natl Acad Sci USA 103:17468-17473

Laemmli UK (1970) Cleavage of structural proteins during assembly of head of bacteriophage-T4. Nature 227:680

Lasker MV, Thai P, Besant PG, Bui CD, Naidu S, Turck CW (2002) Branched-chain 6-ketoacid dehydrogenase kinase: a mammalian enzyme with histidine kinase activity. J Biomol Tech 13: 238-245

Li WW, Heinze J, Haehnel W (2005) Site-specific binding of quinones to proteins through thiol addition and addition-elimination reactions. J Am Chem Soc 127:6140-6141

Marina A, Mott C, Auyzenberg A, Hendrickson WA, Waldburger CD (2001) Structural and mutational analysis of the PhoQ histidine kinase catalytic domain. Insight into the reaction mechanism. J Biol Chem 276:41182-41190. doi:10.1074/jbc.M106080200

Motulsky H, Christopoulos A (2003) Fitting models to biological data using linear and nonlinear regression. A practical guide to curve fitting. GraphPad Software Inc, http://www.graphpad.com, San Diego, CA

Moussatche P, Klee HJ (2004) Autophosphorylation activity of the Arabidopsis ethylene receptor multigene family. J Biol Chem 279:48734-48741

Murakami A, Kim SJ, Fujita Y (1997) Changes in photosystem stoichiometry in response to environmental conditions for cell growth observed with the cyanophyte Synechocystis PCC 6714. Plant Cell Physiol 38:392-397

Panaretou B, Prodromou C, Roe SM, O’Brien R, Ladbury JE, Piper PW, Pearl LH (1998) ATP binding and hydrolysis are essential to the function of the Hsp90 molecular chaperone in vivo. EMBO J 17:4829-4836. doi:10.1093/emboj/17.16.4829

Pfannschmidt T, Nilsson A, Allen JF (1999) Photosynthetic control of chloroplast gene expression. Nature 397:625-628

Plesniak L et al (2002) Probing the nucleotide binding domain of the osmoregulator EnvZ using fluorescent nucleotide derivatives. Biochemistry 41:13876-13882

Puthiyaveetil S, Allen JF (2009) Chloroplast two-component systems: evolution of the link between photosynthesis and gene expression. Proc Royal Soc B 276:2133-2145

Puthiyaveetil S et al (2008) The ancestral symbiont sensor kinase CSK links photosynthesis with gene expression in chloroplasts. Proc Natl Acad Sci USA 105:10061-10066

Puthiyaveetil S, Ibrahim IM, Jelicić B, Tomasić A, Fulgosi H, Allen JF (2010) Transcriptional control of photosynthesis genes: the evolutionarily conserved regulatory mechanism in plastid genome function. Genome Biol Evol 2:888-896. doi:10.1093/ gbe/evq073

Puthiyaveetil S, Ibrahim IM, Allen JF (2013) Evolutionary rewiring: a modified prokaryotic gene regulatory pathway in chloroplasts. Philos T R Soc B 367 doi:10.1098/rstb20120260

Roca J, Wang JC (1992) The capture of a DNA double helix by an ATP-dependent protein clamp: a key step in DNA transport by type II DNA topoisomerases. Cell 71:833-840

Rochaix JD (2007) Role of thylakoid protein kinases in photosynthetic acclimation. FEBS Lett 581:2768-2775

Sardiwal S, Kendall SL, Movahedzadeh F, Rison SC, Stoker NG, Djordjevic S (2005) A GAF domain in the hypoxia/NOinducible Mycobacterium tuberculosis DosS protein binds haem. J Mol Biol 353:929-936

Shimizu M, Kato H, Ogawa T, Kurachi A, Nakagawa Y, Kobayashi H (2010) Sigma factor phosphorylation in the photosynthetic control of photosystem stoichiometry. Proc Natl Acad Sci USA 107:10760-10764

Silverstein T, Cheng L, Allen JF (1993a) Chloroplast thylakoid protein phosphatase reactions are redox-independent and kinetically heterogeneous. FEBS Lett 334:101-105

Silverstein T, Cheng LL, Allen JF (1993b) Redox titration of multiple protein phosphorylations in pea chloroplast thylakoids. Biochim Biophys Acta 1183:215-220

Stewart RC, VanBruggen R, Ellefson DD, Wolfe AJ (1998) TNPATP and TNP-ADP as probes of the nucleotide binding site of $\mathrm{CheA}$, the histidine protein kinase in the chemotaxis signal transduction pathway of Escherichia coli. Biochemistry 37:12269-12279

Stock AM, Robinson VL, Goudreau PN (2000) Two-component signal transduction. Annu Rev Biochem 69:183-215

Thelen JJ, Miernyk JA, Randall DD (2000) Pyruvate dehydrogenase kinase from Arabidopsis thaliana: a protein histidine kinase that phosphorylates serine residues. Biochem J 349:195-201

Trajtenberg F, Grana M, Ruetalo N, Botti H, Buschiazzo A (2010) Structural and enzymatic insights into the ATP binding and autophosphorylation mechanism of a sensor histidine kinase. J Biol Chem 285:24892-24903

Wu JA, Bauer CE (2010) RegB kinase activity is controlled in part by monitoring the ratio of oxidized to reduced ubiquinones in the ubiquinone pool. Mbio 1 